\title{
The Future of the Artificial Pancreas
}

\author{
Hood Thabit, MD, ${ }^{1,2}$ and Roman Hovorka, $\mathrm{PhD}^{1,3}$
}

$\mathbf{I}$ N THIS ISSUE OF DIABETES TECHNOLOGY \& Therapeutics, Wang et al. ${ }^{1}$ evaluated a promising novel fluorescencebased long-term implantable glucose sensor, underlining the potential of diabetes technology to continue improving the management of type 1 diabetes. Insulin pumps and continuous glucose monitoring (CGM) technology have undergone stepwise development and innovation over the past decade, with many takers but relatively few gainers, with the ultimate goal of improving glycemic control and reducing the burden of type 1 diabetes. Insulin pumps enable users to infuse rapidor quick-acting insulin at preselected infusion rates with multiple programmable basal profiles, mimicking basal insulin secretion. Evolution in engineering, information technology, and design have led to patch pumps, sophisticated user interface with data download capacity, cloud connectivity, and integration with CGM. Present-generation CGM sensors are externally worn, measuring subcutaneous glucose in the interstitial space, providing trends and values, generating alarms, having sophisticated data review formats, and ultimately driving insulin delivery. Data from clinical trials and meta-analyses on insulin pump and CGM devices to date have been mixed. ${ }^{2,3}$ When factors associated with reduction in hemoglobin A1c among CGM users were evaluated, ${ }^{4}$ participants who used CGM at least 6 days per week had significantly greater improvement in hemoglobin A1c compared with those using it less often, suggesting benefit in glycemic control mostly among regular users of the devices.

Although insulin pumps and CGM use may be beneficial, these do not remove the complexities of self-managing insulin therapy or the day-to-day variability of glucose levels faced by many people with diabetes. Closed-loop systems, also known as the artificial or bionic pancreas, represent a step toward an autonomous, automated, glucose-responsive insulin delivery system. A closed-loop system couples subcutaneous CGM and subcutaneous insulin pump delivery and differs from conventional pump therapy by the use of a control algorithm that directs insulin delivery based on realtime sensor glucose levels. ${ }^{5}$ In contrast to low glucose suspend (LGS) pumps and predictive low glucose management (PLGM), closed-loop systems modulate insulin delivery below and above the preset pump regimen, thereby addressing both hypo- and hyperglycemia events.
The number of studies involving closed-loop systems has increased over the past decade, characterized by progress from "bench to bedside." ${ }^{-9}$ Glycemic control and hypoglycemia reduction were shown in inpatient studies to be superior to those by conventional therapy, setting the path toward testing closed-loop systems outside of the research facility environment. The first transitional setting studies were performed in diabetes camps and hotels under close supervision and remote monitoring. ${ }^{10,11}$ The reported outcomes, in terms of efficacy and hypoglycemia reduction, were encouraging and comparable to those observed during studies in research facility settings. Thus the next step forward in preparing closed-loop application for potential use in clinical practice was to evaluate its performance and usability without direct supervision or remote monitoring. This was shown to be feasible in a 3-month application of closed loop in real-world free-living conditions, with significant improvements in glycemic control and reduction of hypoglycemia events. ${ }^{12}$ Clinical trials of dual-hormone closed-loop systems using both insulin and glucagon are ongoing, and results from research facility and short-term outpatient studies to date are promising. ${ }^{13,14}$ Further reduction of hypoglycemia with similar mean sensor glucose levels has been reported, compared with insulin-alone closed-loop systems. ${ }^{15,16}$

Although evidence supporting the efficacy and feasibility for closed-loop use is growing, there are several limitations that need to be addressed to support wider adoption by people with type 1 diabetes. Daytime glucose control by closed loop is hampered by immediate postmeal hyperglycemia and postexercise hypoglycemia, because of the delayed action of currently available rapid-acting insulin analogs. Although the majority of closed-loop users during unsupervised home studies were satisfied with their glycemic control and safety provided by the system, both adult and adolescent users were dissatisfied with the size of devices, as well as CGMrelated alarms and calibration needs. ${ }^{17,18}$ Device burden and the aforementioned CGM-specific issues are widely reported to be barriers to adoption of diabetes technology in clinical practice. Therefore for closed-loop systems to be widely accepted, these technical challenges will need to be addressed.

\footnotetext{
${ }^{1}$ Wellcome Trust-MRC Institute of Metabolic Science, University of Cambridge, Cambridge, United Kingdom.

${ }^{2}$ Department of Diabetes \& Endocrinology, Cambridge University Hospitals NHS Foundation Trust, Cambridge, United Kingdom.

${ }^{3}$ Department of Paediatrics, University of Cambridge, Cambridge, United Kingdom.
} 
Wang et al. ${ }^{1}$ in this issue of Diabetes Technology \& Therapeutics evaluated the accuracy and overnight performance of a long-term implantable CGM device. The authors reported a mean sensor life span of 87 days, the longest reported continuous use of a real-time glucose monitoring sensor to date in humans. Using participants' capillary glucose measurements during home use as reference, the implanted sensor had a reported accuracy, as measured by mean absolute relative difference (MARD), of $12.3 \pm 0.7 \%$ over the range of $40-400 \mathrm{mg} / \mathrm{dL}$. This is comparable to or lower than the MARD of other commercially available CGM sensors, which, in contrast, are externally worn and need replacement every 5-7 days. ${ }^{19}$ The accuracy was shown to be consistent over the life of the sensor, which is assuring for long-term sensor use. The incidence of false-positive alarms overnight was very low (4\% of 100 nights when hypoglycemic alarms were activated). This may potentially translate to reduced alarm fatigue, which is known to adversely affect conventional subcutaneous CGM use, thereby increasing benefits gained from regular CGM use.

How will the reported results potentially benefit closedloop system use specifically? Conventional transcutaneous CGM performance can be hindered by sensor signal attenuation, which is prevalent overnight. This is thought to be induced by increased local pressure over the sensor when users lie on it, also known as pressure-induced sensor attenuation (PISA). ${ }^{20}$ This decrease in signal level is not only falsely interpreted as low glucose values by closed-loop system algorithms, it may also adversely affect the performance of currently available LGS and PLGM pump devices, which would erroneously shut-off insulin delivery. In the study by Wang et al., ${ }^{1}$ when pressure was applied by the investigators over the implantable sensor to induce PISA, the outcome of the analysis suggested no evidence of sensor signal attenuation. A separate study assessed the sensor accuracy following 28 days of use during in-clinic visits with the YSI reference and reported MARD of $11.6 \pm 0.7 \%$ for glucose values above $75 \mathrm{mg} / \mathrm{dL}^{21}$ This is of particular relevance for closed-loop systems, which actively modulate insulin delivery within the euglycemic and hyperglycemic ranges.

Current closed-loop use is still influenced by the user's perception of device burden and physical appearances. ${ }^{17,18}$ An implantable sensor may therefore offer a unique solution, given the appeal and perceived benefits of a sensor not externally visible. By reducing the burden of frequent sensor changes and the number of consumables needed, especially when traveling, it will be interesting to speculate on the positive effects this may have on a user's experience and adherence, which are among the limiting factors of conventional subcutaneous sensors. However, considerations will also need to be given to potential drawbacks such as additional clinic visits for sensor implantation and removal (currently approximately once every 90 days), as well as the minimally visible incisional scar. Appropriately designed user evaluation studies may help to inform acceptance, including whether 90 days of sensor durability is sufficient.

The next question therefore is what are the characteristics needed for an ideal glucose sensor for use in closedloop systems in the future. As previously mentioned, having sensors with high and consistent accuracy within the euglycemic-hyperglycemic range is important during closedloop operation, to avoid hypoglycemia from sensor overreading. High hypoglycemia alarm sensitivity and specificity improve compliance and minimize hypoglycemia exposure. Although modern CGM devices have excellent and acceptable accuracy during home use, few have reported on its accuracy and performance during prolonged closed-loop home studies. ${ }^{22,23}$ By doing so, researchers could potentially identify and develop strategies to mitigate against issues related to CGM performance during real-world use of closed loop, which may not be evident in controlled environments or in silico settings. CGM sensors with prolonged life and consistent accuracy may enhance closed-loop wear and outcomes by reducing the need for sensor changes, thereby reducing variability of performance between sensors and inconvenience.

Limiting or eliminating completely sensor signal attenuation, as has been shown in the present study by Wang et al., could potentially avoid erroneous insulin delivery suspension and aberrancy, which may lead to unintended hyperglycemia or ketonemia.

From the user's perspective, CGM systems with low false alarm rates and needing little or no calibration would ensure less frustration and higher CGM use compliance, leading to potentially consistent closed-loop application. At present, the aforementioned implantable sensor, which has reportedly low false alarm rates, fits the former criterion, whereas the recently introduced flash glucose monitoring system, which is factory-calibrated, fits the latter criterion. ${ }^{24}$

CGM sensors for closed-loop application should ideally have all the features described above, combined into one device.

These past few years have certainly been exciting times for closed-loop research. Technological and pharmacological advancements will hopefully lead to better sensors and faster insulins being developed. The ultimate goal is that the optimism and potential of closed-loop technology would become a reality for people with type 1 diabetes in the near future. Although challenges are still there, recent developments have shown that the outlook does look promising.

\section{References}

1. Wang X, Ioacara S, DeHennis A: Long-term home study on nocturnal hypoglycemic alarms using a new fully implantable continuous glucose monitoring system in type 1 diabetes. Diabetes Technol Ther 2015;17:780-786.

2. Juvenile Diabetes Research Foundation Continuous Glucose Monitoring Study Group: Effectiveness of continuous glucose monitoring in a clinical care environment: evidence from the Juvenile Diabetes Research Foundation continuous glucose monitoring (JDRF-CGM) trial. Diabetes Care 2010;33:17-22.

3. Yeh HC, Brown TT, Maruthur N, et al.: Comparative effectiveness and safety of methods of insulin delivery and glucose monitoring for diabetes mellitus: a systematic review and meta-analysis. Ann Intern Med 2012;157:336347.

4. Fiallo-Scharer R, Cheng J, Beck RW, et al.: Factors predictive of severe hypoglycemia in type 1 diabetes: analysis from the Juvenile Diabetes Research Foundation continuous glucose monitoring randomized control trial dataset. Diabetes Care 2011;34:586-590. 
5. Hovorka R: Closed-loop insulin delivery: from bench to clinical practice. Nat Rev Endocrinol 2011;7:385-395.

6. Cobelli C, Renard E, Kovatchev B: Artificial pancreas: past, present, future. Diabetes 2011;60:2672-2682.

7. Lee SW, Welsh JB: Upcoming devices for diabetes management: the artificial pancreas as the hallmark device. Diabetes Technol Ther 2015;17:538-541.

8. Peyser T, Dassau E, Breton M, et al.: The artificial pancreas: current status and future prospects in the management of diabetes. Ann N Y Acad Sci 2014;1311:102-123.

9. Shah VN, Shoskes A, Tawfik B, et al.: Closed-loop system in the management of diabetes: past, present, and future. Diabetes Technol Ther 2014;16:477-490.

10. Phillip M, Battelino T, Atlas E, et al.: Nocturnal glucose control with an artificial pancreas at a diabetes camp. $\mathrm{N}$ Engl J Med 2013;368:824-833.

11. Kovatchev BP, Renard E, Cobelli C, et al.: Feasibility of outpatient fully integrated closed-loop control: first studies of wearable artificial pancreas. Diabetes Care 2013;36: 1851-1858.

12. Thabit H, Tauschmann M, Allen JM, et al.: Home use of an artificial beta cell in type 1 diabetes. N Engl J Med 2015 September 17 [Epub ahead of print] doi: 10.1056/NEJMoa1509351.

13. Russell SJ, El-Khatib FH, Nathan DM, et al.: Blood glucose control in type 1 diabetes with a bihormonal bionic endocrine pancreas. Diabetes Care 2012;35:2148-2155.

14. Russell SJ, El-Khatib FH, Sinha M, et al.: Outpatient glycemic control with a bionic pancreas in type 1 diabetes. $\mathrm{N}$ Engl J Med 2014;371:313-325.

15. Haidar A, Legault L, Messier V, et al.: Comparison of dualhormone artificial pancreas, single-hormone artificial pancreas, and conventional insulin pump therapy for glycaemic control in patients with type 1 diabetes: an open-label randomised controlled crossover trial. Lancet Diabetes Endocrinol 2015;3:17-26.

16. Haidar A, Legault L, Matteau-Pelletier L, et al.: Outpatient overnight glucose control with dual-hormone artificial pancreas, single-hormone artificial pancreas, or conventional insulin pump therapy in children and adolescents with type 1 diabetes: an open-label, randomised controlled trial. Lancet Diabetes Endocrinol 2015;3:595-604.
17. Barnard KD, Wysocki T, Allen JM, et al.: Closing the loop overnight at home setting: psychosocial impact for adolescents with type 1 diabetes and their parents. BMJ Open Diabetes Res Care 2014;2:e00025.

18. Barnard KD, Wysocki T, Thabit $\mathrm{H}$, et al.: Psychosocial aspects of closed- and open-loop insulin delivery: closing the loop in adults with type 1 diabetes in the home setting. Diabet Med 2015;32:601-608.

19. Damiano ER, McKeon K, El-Khatib FH, et al.: A comparative effectiveness analysis of three continuous glucose monitors: the Navigator, G4 Platinum, and Enlite. J Diabetes Sci Technol 2014;8:699-708.

20. Bequette BW: Challenges and recent progress in the development of a closed-loop artificial pancreas. Annu Rev Control 2012;36:255-266.

21. Mortellaro M, DeHennis A: Performance characterization of an abiotic and fluorescent-based continuous glucose monitoring system in patients with type 1 diabetes. Biosens Bioelectron 2014;61:227-231.

22. Bailey TS, Chang A, Christiansen M: Clinical accuracy of a continuous glucose monitoring system with an advanced algorithm. J Diabetes Sci Technol 2015;9:209-214.

23. Thabit H, Leelarathna L, Wilinska ME, et al.: Accuracy of continuous glucose monitoring during three closed-loop home studies under free-living conditions. Diabetes Technol Ther 2015; 14:801-807.

24. Bailey T, Bode BW, Christiansen MP, et al.: The performance and usability of a factory-calibrated flash glucose monitoring system. Diabetes Technol Ther 2015;17:787794.

Address correspondence to: Roman Hovorka, PhD

University of Cambridge Metabolic Research Laboratories and NIHR Cambridge Biomedical Research Centre Wellcome Trust-MRC Institute of Metabolic Science Box 289

Addenbrooke's Hospital Hills Road Cambridge CB2 OQQ, United Kingdom

E-mail:rh347@cam.ac.uk 